\title{
Hypoxia and extracellular matrix proteins influence angiogenesis and lymphangiogenesis in mouse embryoid bodies
}

\section{Andrea M. Foskett ${ }^{1}$, Uthayashanker R. Ezekiel ${ }^{2}$, Jerome P. Trzeciakowski ${ }^{1}$, David C. Zawieja ${ }^{1}$ and Mariappan Muthuchamy ${ }^{1 *}$}

Division of Lymphatic Biology, Department of Systems Biology and Translational Medicine, Texas A\&M Health Science Center College of Medicine, Cardiovascular Research Institute, College Station, TX, USA

${ }^{2}$ Clinical Laboratory Science, School of Allied Health, Saint Louis University Medical Center, Saint Louis, MO, USA

\section{Edited by:}

Agustín Guerrero-Hernández,

CINVESTAV, Mexico

Reviewed by:

Ricardo Espinosa-Tanguma

Universidad Autónoma de San Luis

Potosi, Mexico

Jose R. Lopez, University of

Valladolid, Spain

Bruno Escalante, CINVESTAV, Mexico

*Correspondence:

Mariappan Muthuchamy, Department of Systems Biology and Translational Medicine, Texas A\&M Health Science Center College of Medicine, 336 Reynolds Medical Building, College Station, TX 77843-1114, USA e-mail:marim@tamu.edu
Regulatory mechanisms for angiogenesis are relatively well established compared to lymphangiogenesis. Few studies have shown that a combination of vascular endothelial growth factor VEGF-A/C with hypoxia or collagen matrix promotes lymphatic structures along with blood vessel development in mouse embryoid bodies (EB). In this study we tested the hypothesis that while hypoxia combined with prolonged VEGF-A/C treatment would induce early lymphangiogenesis in addition to angiogenesis in mouse EBs, under similar conditions specific extracellular matrix (ECM) proteins would promote lymphatic vessel-like structures over angiogenesis. EBs were subjected to four conditions and were maintained under normoxia and hypoxia ( $21 \%$ and $2.6 \% \mathrm{O}_{2}$, respectively) with or without VEGF-A/C. Microarray analyses of normoxic and hypoxic EBs, and immunofluorescence data showed very low expression of early lymphatic endothelial cell (LEC) markers, lymphatic vessel endothelial hyaluronan receptor 1 (LYVE1), and prospero-related homeobox 1 (Prox1) at early time points. Double immunofluorescence using MECA-32 and Prox1/LYVE1 demonstrated that combined hypoxia and VEGF-A/C treatment promoted formation of blood vessel-like structures, whereas only Prox $1^{+} /$LYVE1 ${ }^{+}$LECs were detected in EBs at E22.5. Furthermore, EBs were grown on laminin or collagen-I coated plates and were subjected to the four treatments as described above. Results revealed that LECs in EBs at E36.5 attached better to collagen-l, resulting in an organized network of lymphatic vessel-like structures as compared to EBs grown on laminin. However, blood vessel-like structures were less favored under these same conditions. Collectively, our data demonstrate that hypoxia combined with growth factors promotes angiogenesis, whereas combination of these conditions with specific ECM proteins favors lymphangiogenesis processes in mouse EBs.

Keywords: angiogenesis, lymphangiogenesis, embryoid body, hypoxia, VEGF

\section{INTRODUCTION}

The lymphatic system is a specialized vasculature that is intimately involved in body fluid circulation, macromolecular homeostasis, lipid absorption, and immune function. Interstitial fluid enters via lymphatic capillaries and is transported into the venous circulation via larger collecting lymphatics, which are comprised of muscle and adventitial layers wrapped around the endothelial tube (Schmid-Schonbein, 1990; Borisov, 2005). Lymphatic muscle exhibits important differences from typical vascular smooth muscle that are critical to overall lymphatic function (Muthuchamy et al., 2003). The phasic and tonic contractility of lymphatic muscle function is essential for lymph propulsion and transport (Gashev et al., 2002, 2004). A dysfunctional lymphatic circulation can result in a wide range of clinical disorders including edema, altered lymphocyte circulation, depressed immune function, impaired lipid metabolism, etc. Primary impairment of lymphatic function produces lymphedema, which can be the result of numerous known pathologies such as lymphatic filariasis, post surgical resection of lymph nodes and lymphatics, and inflammatory processes. Though several studies have unraveled the mechanisms by which lymphangiogenesis promotes new lymphatic growth in tissues with lymphedema, there are few efficacious therapies, as well as a nearly complete lack of medical treatment options for lymphatic dysfunction (Szuba and Rockson, 1998; Rockson, 2006). In large part, this is due to the relatively poor understanding of the mechanisms of lymphatic vessel development, particularly lymphangiogenesis and muscle cell recruitment processes, and lymphatic vessel functions.

In the last decade, several studies have elucidated key genes involved in different stages of embryonic lymphangiogenesis in mammals (Oliver, 2004; Oliver and Alitalo, 2005), including lymphatic vessel endothelial hyaluronan receptor 1 (LYVE1), transcription factor prospero-related homeobox 1 (Prox1), and vascular endothelial growth factor receptor 3 (VEGFR-3) amongst several others (Kaipainen et al., 1995; Banerji et al., 1999; Wigle and Oliver, 1999; Hong et al., 2002; Wigle et al., 2002). LYVE1 
contributes to lymphatic endothelial cell (LEC) competence and its embryologic expression is observed around E9.0-E9.5 (Banerji et al., 1999). Prox1 (Wigle and Oliver, 1999; Hong et al., 2002; Wigle et al., 2002), which is expressed at E9.5-E10.5, is the master regulator of LEC fate and is critical to lymphatic bias. In addition, lymphatic specification occurs at E12.5 days (Oliver, 2004; Oliver and Alitalo, 2005); during which LEC differentiation is detected along with the expression of VEGFR-3 (Kaipainen et al., 1995; Dumont et al., 1998). Furthermore additional regulatory molecules, such as Podoplanin (Schacht et al., 2003; Navarro et al., 2008), neuropilin-2 (Yuan et al., 2002) angiopoietin-2 (Gale et al., 2002; Veikkola and Alitalo, 2002), and ephrinB2 (Adams et al., 1999; Makinen et al., 2005) have been shown to be involved in the development of early and postnatal lymphatic vasculature.

In spite of the importance of these several molecular markers for early lymphatic vessel development, the molecular mechanisms that regulate this complex process are not yet completely understood. Gene knockout studies on several lymphatic endothelial markers (Jeltsch et al., 1997; Dumont et al., 1998; Karkkainen et al., 2004) revealed the necessity of these genes in early lymphatic endothelial differentiation and vessel development but failed to provide a model to study their regulatory mechanisms since most of the targeted mice are either embryonically or perinatally lethal. To overcome the problems associated with knockout models, several studies addressed the molecular mechanisms of early developmental processes by comparing the differentiation of mouse embryonic stem (ES) cell-derived embryoid bodies (EB) from wild type and knockout ES cell lines. Liersch et al. (2006) demonstrated that in the absence of VEGF-A/C, normoxic E27-day-old EBs developed both blood and lymphatic structures. However, adding VEGF-A or VEGF-C to the media potently promoted lymphatic vessel formation. Kreuger et al. (2006) established that normoxic E18-day-old EBs cultured in 3D collagen matrices and supplemented with VEGF-A/C formed lymphatic vessel-like structures, and that hypoxia had little or no effect on the development of early lymphatics. Recently Cueni et al. (2010) emphasized the role for podoplanin in inhibiting lymph vessel formation in E22day-old mouse EBs grown in the presence of VEGF-A/C. These data indicate that the mouse EB model could recapitulate early stages of lymphatic vessel formation given the right environmental triggers, such as growth factors and extracellular matrix (ECM) proteins. Taken together, we propose that while prolonged VEGF$\mathrm{A} / \mathrm{C}$ treatment would induce both early lymphangiogenesis and angiogenesis in mouse EBs, other environmental cues like ECM proteins would differentially promote lymphatic or blood vessel formation.

In the present study, we first performed microarray analyses in developing EBs to compare the gene expression patterns that occurred during the processes of angiogenesis and lymphangiogenesis. Then we tested the combined effects of oxygen treatments, presence or absence of VEGF-A/C, and the presence or absence of ECM proteins (collagen-I and laminin) in promoting the formation of blood or lymphatic vessel-like structures in mouse EBs.

\section{MATERIALS AND METHODS CELL CULTURE AND ESTABLISHMENT OF MOUSE EMBRYOID BODY MODEL}

Embryonic stem cells derived from 129/SvJ mice were cultured on a feeder layer of mitotically inactivated primary mouse embryonic fibroblasts, as we have described previously (Ezekiel, 2007). The ES cells were maintained in an undifferentiated state by supplementing the ES media with leukemia inhibitory factor (LIF; Chemicon International, Temecula, CA, USA). After three passages, ES cell differentiation was achieved by the removal of fibroblasts and LIF. A schematic of the EB model is illustrated in Figure 1. At E3.5, EBs were maintained in normoxia $\left(21 \% \mathrm{O}_{2}\right)$ in $100 \mathrm{~mm}$ bacterial culture plates (Corning Incorporated, Corning, NY, USA)
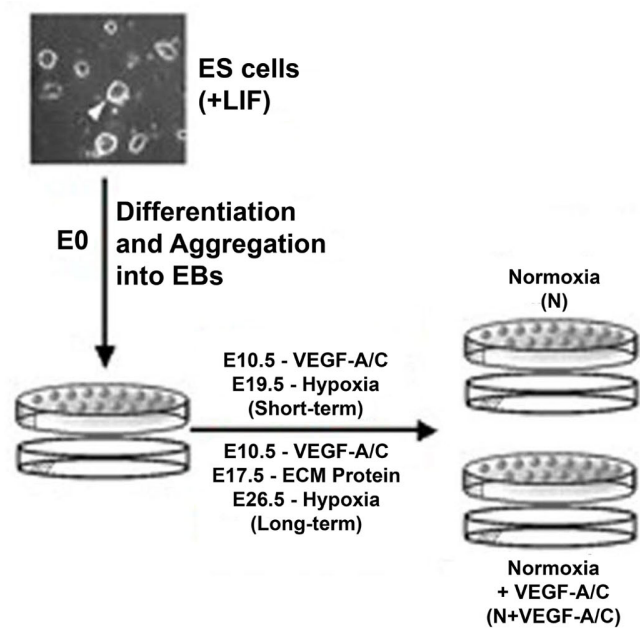

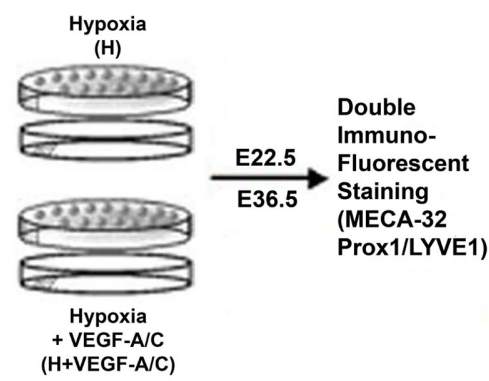

FIGURE 1 | Schematic illustration of the embryoid body (EB) model. The ES cell and EB culture conditions, and the durations of growth factors and hypoxic conditions have been described in the Section "Materials and Methods." EBs were maintained in normoxia at $21 \% \mathrm{O}_{2}$ and hypoxic EBs were maintained at $2.6 \% \mathrm{O}_{2}$. 
and cultured with Dulbecco modified Eagle medium (DMEM; Gibco, Eggenstein, Germany) containing 4,500 mg/L D-glucose, Lglutamine, pyridoxine hydrochloride, and $25 \mathrm{mM}$ HEPES buffer. DMEM was additionally supplemented with 15\% fetal bovine serum (FBS; Gibco), $1.2 \mathrm{mM}$ sodium pyruvate (Gibco), 0.12\% monothioglycerol (Sigma, St. Louis, Missouri), and 1\% antibiotic/antimycotic (Gibco). For the microarray and real-time PCR experiments (scheme not shown in Figure 1), EBs were transferred on days E8, E12, E15, and E18 to hypoxia $\left(2.6 \% \mathrm{O}_{2}\right)$ or maintained in normoxia. After 2 days of differential oxygen treatments, these EBs were analyzed at E10, E14, E17, and E20 respectively. Hypoxia was achieved in a humidified chamber flushed with a mixture of $95 \% \mathrm{~N}_{2} / 5 \% \mathrm{CO}_{2}$ gas and room air until the oxygen level was stably maintained at $2.6 \%$. For the immunohistochemistry (IHC) experiments (scheme shown in Figure 1), at E10.5, EBs were transferred to $35 \mathrm{~mm}$ plates and half the EBs was maintained in media supplemented with recombinant human VEGF-A (R\&D Systems, Minneapolis, MN, USA; $20 \mathrm{ng} / \mathrm{ml}$ ) and VEGF-C (R\&D Systems; $100 \mathrm{ng} / \mathrm{ml}$ ). To test the effects of short-term hypoxia, some EBs with/without VEGF-A/C was transferred to hypoxia on E19.5 and analyzed at E22.5. For long-term hypoxia experiments, some EBs received VEGF-A/C at E10.5, further all EBs at E17.5 were seeded onto a layer of either collagen-I (Sigma; $50 \mu \mathrm{g} / \mathrm{ml}$ ) or laminin (Sigma; $20 \mu \mathrm{g} / \mathrm{ml}$ ). Thereafter some EBs with/without VEGF-A/C was transferred to hypoxia on E26.5 and analysis was performed at E36.5.

\section{DOUBLE IMMUNOFLUORESCENCE AND MICROSCOPY}

Embryoid bodies cultures were fixed with $2 \%$ paraformaldehyde (Sigma) for $1 \mathrm{~h}$ at room temperature (RT) followed by permeabilization with ice-cold methanol for $5 \mathrm{~min}$ at $4^{\circ} \mathrm{C}$. EB samples were then incubated with blocking solution containing 1\% BSA (Vector Laboratories, UK) and 5\% Normal Goat Serum (RDI, Concord, MA, USA) for $1 \mathrm{~h} / \mathrm{RT}$. These were treated with primary antibodies (MECA-32, BD Biosciences; Prox1, Abcam, Cambridge, MA, USA; LYVE1, Abcam) and appropriate secondary antibodies (Oregon Green 488, Molecular Probes, Invitrogen; Cy5, Abcam). Samples were mounted onto glass slides using Prolong antifade solution (Molecular Probes, Invitrogen). Average projections were produced from a 3D series of sections, where each $0.5 \mu \mathrm{m}$ thick slice was imaged using the Leica AOBS SP2 Confocal microscope with either a U APO $40 \times$ water objective of numerical aperture 1.15 or a $20 \times$ dry objective of numerical aperture 0.75 . Each experiment set was repeated three times for the set of marker genes and we sampled various regions of EBs from different replicates within a condition.

\section{RNA ISOLATION}

Total RNA was isolated from EBs (E14, E17, E20) using RNA STAT60 (Tel-Test Inc., Friendswood, TX, USA). Briefly, cells were lysed in RNA STAT-60 by repetitive pipetting. Then chloroform was added to separate the lysate into an aqueous phase containing exclusively the total RNA and an organic phase containing DNA and other proteins. The total RNA was precipitated out by the addition of isopropanol, further washed with ethanol and solubilized in water. The RNA was subsequently DNase treated with DNAfree $^{\mathrm{TM}}$ (Ambion, Austin, TX, USA) and analyzed for both quality and quantity using the Agilent 2100 Bioanalyzer and RNA6000 Nano LabChip Kit (Agilent Technologies).

\section{REAL-TIME POLYMERASE CHAIN REACTION (RT-PCR)}

CDNA was synthesized using the iScript cDNA synthesis kit (BioRad, Hercules, CA, USA). PCR primers for MECA-32, Prox1, LYVE1, VEGFR-3, and GAPDH were designed using the Beacon Designer v6.0 software (PREMIER Biosoft International, Palo Alto, CA, USA) and all primers were ordered from Sigma Genosys. cDNA of Universal Mouse Reference RNA (UMRR) and EB samples were mixed with appropriate primers and SYBR Green supermix (BioRad) and amplified using the iCycler iQ Real-Time PCR Detection System (BioRad). Quantification of the real-time PCR data was performed using the delta-delta $\mathrm{Ct}(\Delta \Delta \mathrm{Ct})$ model. In this model, the quantity of each RNA transcript in each EB sample was compared to that of GAPDH in the same sample to obtain $\Delta \mathrm{Ct}$ values. These values were then normalized relative to a reference sample, Universal Mouse Reference RNA (UMRR; Stratagene, La Jolla, CA, USA) to obtain $\Delta \Delta$ Ct values. Experiments were performed four times for statistical analysis.

\section{BOOTSTRAP STATISTICS}

Gene expression fold changes were compared using permutation tests (Hyde et al., 2007), in which, data from each gene were resampled randomly with replacement, without regard to type of oxygen treatment. Sample and calibrator values for both target and reference $(\mathrm{GAPDH})$ genes were permuted independently to permit the variance of both technical and biological replicates to be expressed in the results. Following each reordering of the data, the ratio of fold changes was calculated in the same manner as was used for the unordered data, and the results stored to form a new distribution of 1000 resampled ratios. The fraction of the permuted distribution whose values exceeded the original (non-permuted) N/H ratio was then used to compute the probability that the observed $\mathrm{N} / \mathrm{H}$ ratio occurred by chance. Permutation tests are considered superior to ANOVA or $t$-tests for small samples, or when sample distributions are non-normal, as they entail no assumptions regarding the form of the statistical distribution. Calculations were performed on data in the form of $\mathrm{N} / \mathrm{H}$ fold-increase ratios. Comparisons of $p$-values between tests using fold-increase ratios for the four genes over the three embryonic days were highly correlated $(r=0.999)$, indicating the test results were not affected by the method of calculation or the shape of the sample distribution. All data resampling operations were performed using the S-PLUS programming language, v. 7.02 (Insightful Corp., Seattle, WA, USA).

\section{MICROARRAY}

Total RNA was amplified using the Amino Allyl MessageAmp aRNA kit (Ambion). The aRNA amplification procedure begins with first strand cDNA synthesis where total RNA is reverse transcribed using an oligo(dT) primer containing a T7 RNA polymerase promoter sequence. The resulting cDNA then undergoes second strand synthesis serving as a template for in vitro transcription, which generates hundreds of antisense RNA (aRNA) with incorporated modified nucleotide 5-(3-aminoallyl)-UTP (aaUTP). The yield and quality of aRNA is assessed using the Agilent 2100 Bioanalyzer (Agilent Technologies). The aRNA is then 
chemically dye-coupled to NHS esters of CY5 dye (Amersham Biosciences, Piscataway, NJ, USA). UMRR (Stratagene, La Jolla, CA, USA) was amplified in a similar manner and labeled with NHS ester-Cy3 (Amersham Biosciences). The dye-coupled RNA samples were hybridized to glass arrays containing the Operon Mouse Genome Oligo Set Version 3.0 containing $36 \mathrm{~K}$ oligos (fabricated in our departmental core lab). Following hybridization, the arrays were scanned at $532 \mathrm{~nm}$ and $635 \mathrm{~nm}$ wavelengths with a $10 \mu \mathrm{m}$ resolution using a GenePix 4000A scanner (Axon Instruments). The two individual wavelength scans are then integrated and superimposed into a color ratio image (Red vs. Green). The acquired raw data was transformed, normalized and filtered using GeneSpring GX v7.3.1 software (Agilent Technologies). Differential gene expression was assessed using Welsh ANOVAs and twosample $t$-tests (false discovery rate $<0.05$ ) followed by a two-fold change restriction.

\section{RESULTS \\ EFFECTS OF SHORT-TERM HYPOXIA ON GENE EXPRESSION PROFILES OF MOUSE EBS AT DIFFERENT DAYS OF EMBRYONIC DEVELOPMENT}

The data discussed in this publication have been deposited in NCBI's Gene Expression Omnibus and are accessible through GEO Series accession number GSE17613 (http://www.ncbi.nlm.nih.gov/geo/query/acc.cgi?acc = GSE17613). Microarray data from the EBs under normoxic and hypoxic conditions identified a total of 357 and 995 genes, respectively that were significantly up- or down-regulated $(p<0.05$ by at least twofold across any of the time points E10, E14, E17, and E20. Selective genes from the total of 357 and 995 genes are listed in Table 1 and 2 , respectively. These include a host of growth factors, transcription factors, and contractile proteins associated with angiogenesis and cardiogenesis. Genes that respond to hypoxic conditions, like ERO-1 was upregulated with hypoxic treatment, indicating that the conditions we employed provided a hypoxic environment. In addition, several set of genes that are related to cell growth, development and cell-cell signaling were differentially regulated in response to both oxygen treatments (data not shown). In this study, we focused on the angiogenesis and lymphangiogenesis processes in developing EBs.

Vascular endothelial growth factor receptor 1 (VEGFR-1) and vascular endothelial growth factor A (VEGF-A) were upregulated in EBs subjected to hypoxia between E10 and E20 (Table 2). Furthermore differential expression patterns of contractile proteins is observed between hypoxic and normoxic EBs (Tables 1 and 2). However, differences in lymphatic markers and genes responsible for early lymphatic vessel formation such as Prox1, LYVE1, VEGFR-3, Tie2, Podoplanin, and those involved in vessel/network maturation and valve formation such as Syk/SLP76 and FoxC2 were not statistically significant in either oxygen treatments. The raw normalized values for these genes from the microarray data have been reported in Table 3. Quantitative-PCR (qPCR) analyses of gene expression for selective LEC markers, including LYVE1, VEGFR-3, and Prox1, and blood endothelial cell (BEC) marker, MECA-32 during EB development under normoxic and hypoxic conditions are shown in Figure 2. Results demonstrate that both blood and lymphatic marker genes are significantly upregulated under normoxic conditions compared to hypoxic conditions.
Figure 3A shows positive staining for both BECs and LECs in normoxic (N) EBs. Hypoxic EBs formed MECA-32 ${ }^{+}$blood vessel-like structures that were usually densely localized in one small region within a single EB (Figure 3B). In addition early LEC differentiation (Prox1 ${ }^{+}$cells) was detected in hypoxic EBs in close vicinity to these blood vessel-like structures (arrowheads in Figure 3B).

\section{STIMULATION WITH VEGF-A/C PREFERENTIALLY PROMOTES FORMATION OF BLOOD VESSEL-LIKE STRUCTURES IN E22.5-DAY-OLD} EBs

Figure 4 shows the light microscope images of E22.5-day-old EBs under VEGF-A/C-treated normoxic (N + VEGF-A/C) and VEGFA/C-treated hypoxic $(\mathrm{H}+\mathrm{VEGF}-\mathrm{A} / \mathrm{C})$ conditions as described in the Section "Materials and Methods." EB cultures revealed extension and contact of adjacent EBs to form cord-like structures, indicated by arrowheads in Figure 4. Furthermore under both conditions, N + VEGF-A/C (Figure 5A) and H + VEGF-A/C, long isolated MECA-32 ${ }^{+}$blood vessel-like structures (marked by arrowheads in Figure 5B) that spanned several EBs were clearly detected. In addition no differences were seen in the patterns of LEC differentiation between EB cultures grown with or without VEGF-A/C, either under normoxic or hypoxic conditions (Figures 3 and 5). However, in all treatments except normoxia, it must be noted that Prox $1^{+}$LECs were predominantly found surrounding regions where blood vessel-like structures were observed. Certain regions of the blood vessel-like structures also stained weakly for Prox1 (Figures 3 and 5).

\section{DIFFERENTIAL ROLES OF EXTRACELLULAR MATRIX PROTEINS IN PROMOTING INDUCTION OF LYMPHATIC VESSEL-LIKE STRUCTURES IN E36.5-DAY-OLD EBs}

Double immunostaining of E36.5-day-old EBs grown on collagenI (Figures 6 and 7) or laminin (Figure 8) was performed with the BEC marker, MECA-32 and one of two LEC markers, Prox1 or LYVE1. Normoxic E36.5 EBs (Figure 6A) were not morphologically different than the previous observations at E22.5. As seen in Figure 6B, E36.5 hypoxic EBs grown on collagen-coated plates promoted some Prox ${ }^{+}$lymphatic vessel-like structures (arrowheads) but failed to promote MECA- $32^{+}$blood vessel-like structures like those observed at E22.5 (Figure 3B). Furthermore $\mathrm{N}+\mathrm{VEGF}-\mathrm{A} / \mathrm{C}$ (Figure 6C) and H + VEGF-A/C (Figure 7) EBs grown on collagen-coated plates developed the most prominent lymphatic vessel-like structures, indicated by arrowheads, with weak staining for MECA-32 ${ }^{+}$BECs also present within these structures.

Embryoid bodies grown on laminin-coated plates under normoxia (Figure 8A) or hypoxia (Figure 8B) exhibited only positive staining for both BECs and LECs. N + VEGF-A/C EBs (Figure 8C) formed ring-like structures, marked by arrowheads and exhibited distinctive staining for LYVE1 and MECA-32. H + VEGF-A/C (Figure 8D) promoted LEC differentiation mostly on the periphery of differentiating EBs with no co-staining for MECA-32 in the same region.

\section{DISCUSSION}

Using the mouse EB model, this present study demonstrates that hypoxia with an appropriate combination of growth factors and/or 
Table 1 | Microarray analysis of selected genes of interest from a total of 357 genes that are at least twofold up- or down-regulated $(p<0.05$; $n=3$ ) across age namely E10, E14, E17, and E20 when subjected to normoxic treatment.

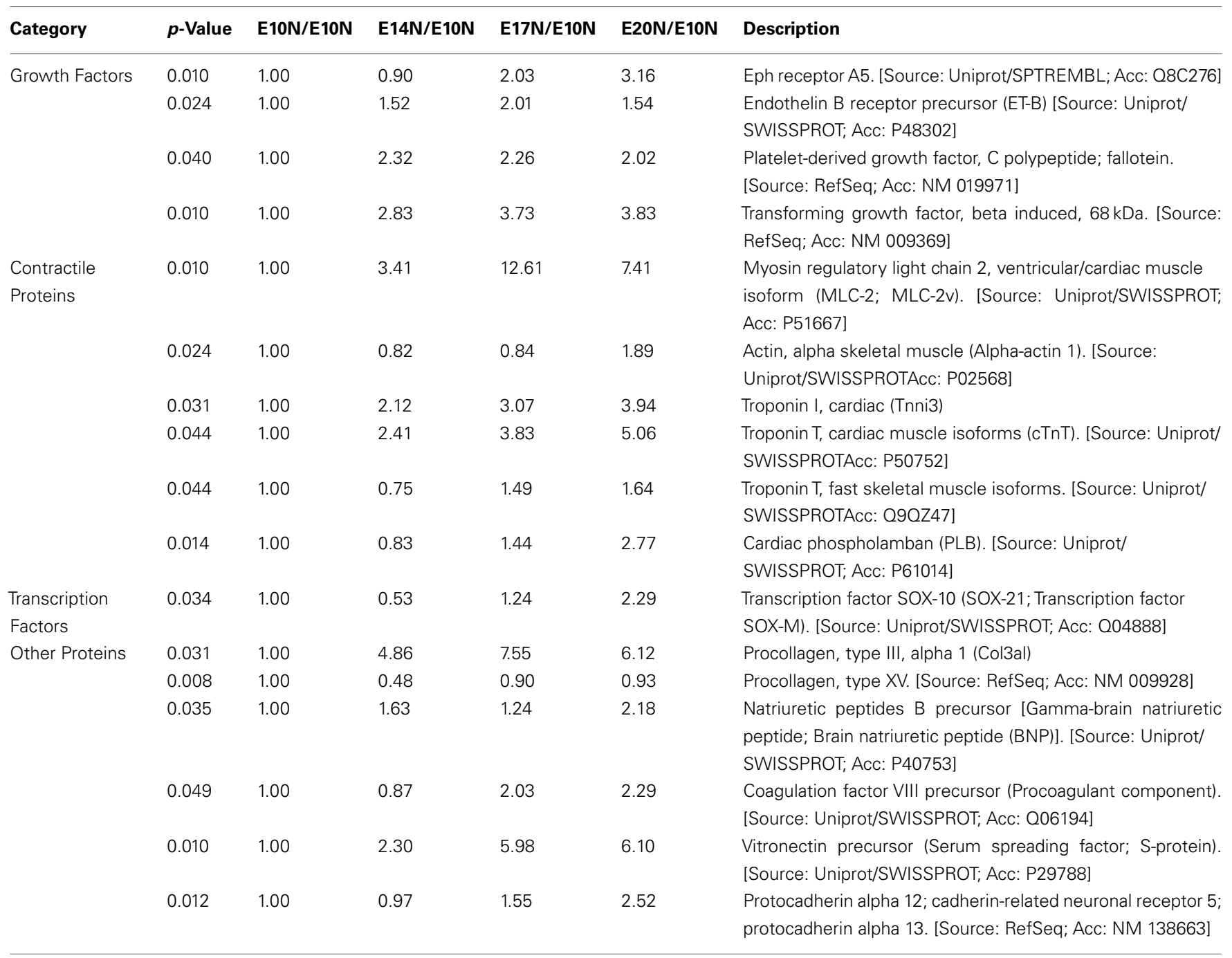

Fold changes across all time points are normalized to E10N.

ECM proteins can induce both blood and lymphatic developmental processes in differentiating EBs. While hypoxia along with VEGF-A/C promotes blood vessel growth at a relatively early time point (E22.5), EBs under similar conditions on a collagen matrix favor lymphatic vessel growth at later time points (E36.5).

It is well known that these developmental processes are very carefully timed and orchestrated in embryonic development with lymphangiogenesis being delayed behind angiogenesis (Sabin, 1902; Oliver and Alitalo, 2005). This concept was well corroborated in the mouse EB model (Figures 3-8), where the data show the presence of predominantly MECA- $32^{+}$blood vessel-like structures at E22.5 and at a later time point (E36.5) prominent lymphatic vessel-like structures $\left(\operatorname{Prox} 1^{+} / \mathrm{LYVE} 1^{+}\right)$are favored. Nilsson et al. (2004) demonstrated that the effects of hypoxia on blood vascular development were most prominent only during a certain stage (E8-E12), and that the primitive vasculature needed sufficient time to develop before it could be remodeled by hypoxia. This serves as a parallel explanation for the organized blood vascular structures and the beginnings of LEC induction at E22.5, followed later by organized lymphatic structures at E36.5. Furthermore, the microarray data for early EB development indicate that while angiogenesis-related marker genes (VEGFR-1 and VEGF-A) are upregulated in hypoxic conditions, lymphangiogenesis-related gene expression is not significantly altered at those time points (Tables 1 and 2). It is possible that similar to development of the blood vasculature, a critical window of time exists when hypoxia could have a positive impact on lymphatic development. The data presented in this study indicate that this discrete stage in lymph vessel development may be confined to later time points in EB development, between E22-E36.

Liersch et al. (2006) tested the effects of growth factors, including VEGF-A and VEGF-C on the development of lymphatic structures in E21 and E27 normoxic EBs, while the effects of 
Table 2 | Microarray analysis of selected genes of interest from a total of 995 genes that are at least twofold up- or down-regulated $(p<0.05$; $n=3$ ) across age namely E10-E20 when subjected to hypoxic treatment.

\begin{tabular}{|c|c|c|c|c|c|c|}
\hline Category & $p$-Value & E10H/E10H & E14H/E10H & E17H/E10H & $\mathrm{E} 20 \mathrm{H} / \mathrm{E} 10 \mathrm{H}$ & Description \\
\hline Hypoxia induced & 0.003 & 1 & 2.22 & 2.26 & 2.40 & EROI-like. [Source: RefSeq; Acc: NM_015774] \\
\hline \multirow[t]{5}{*}{ Growth factors } & 0.028 & 1 & 0.85 & 0.87 & 0.45 & $\begin{array}{l}\text { Vascular endothelial growth factor receptor } 1 \text { precursor } \\
\text { (VEGFR 1; Tyrosine-protein kinase receptor FLT; FLT-1) [Source: } \\
\text { Uniprot/SWISSPROT; Acc: P35969] }\end{array}$ \\
\hline & 0.045 & 1 & 1.59 & 0.93 & 2.08 & $\begin{array}{l}\text { Vascular endothelial growth factor A precursor (VEGF-A; Vascu- } \\
\text { lar permeability factor, VPF). [Source: Uniprot/SWISSPROT; Acc: } \\
\text { Q00731] }\end{array}$ \\
\hline & 0.032 & 1 & 1.94 & 2.04 & 0.97 & $\begin{array}{l}\text { Ephrin type-A receptor } 7 \text { precursor (Tyrosine-protein kinase } \\
\text { receptor EHK-3) [Source: Uniprot/SWISSPROT; Acc: 061772] }\end{array}$ \\
\hline & 0.047 & 1 & 0.70 & 0.43 & 0.59 & $\begin{array}{l}\text { Transforming growth factor beta } 4 \text { precursor (TGF-beta 4; Left } \\
\text { protein) [Source: Uniprot/SWISSPROT; Acc: O64280] }\end{array}$ \\
\hline & 0.036 & 1 & 1.74 & 2.06 & 2.11 & $\begin{array}{l}\text { Insulin-like growth factor II precursor (IGF-II). [Source: Uniprot/ } \\
\text { SWISSPROT; Acc: P09535] }\end{array}$ \\
\hline \multirow{3}{*}{ proteins } & 0.001 & 1 & 1.05 & 1.14 & 2.34 & $\begin{array}{l}\text { Actin, aortic smooth muscle (Alpha-actin 2). [Source: Uniprot/ } \\
\text { SWISSPROT; Acc: P03996] }\end{array}$ \\
\hline & 0.035 & 1 & 3.00 & 4.86 & 6.72 & Actin. alpha cardiac. [Source: Uniprot/SWISSPROT: Acc: P04270] \\
\hline & 0.011 & 1 & 1.05 & 2.97 & 2.52 & $\begin{array}{l}\text { Troponin I, slow skeletal muscle (Troponin I, slow-twitch isoform). } \\
\text { [Source: Uniprot/SWISSPROT; Acc: Q9WUZ5] }\end{array}$ \\
\hline $\begin{array}{l}\text { Transcription } \\
\text { factors }\end{array}$ & 0.034 & 1 & 1.71 & 1.17 & 0.78 & $\begin{array}{l}\text { Neurogenic locus notch homolog protein } 4 \text { precursor (Notch 4) } \\
\text { [Source: Uniprot/SWISSPROT; Acc: P31695] }\end{array}$ \\
\hline \multirow[t]{2}{*}{$\begin{array}{l}\text { Cytokines/ } \\
\text { chemokines }\end{array}$} & 0.034 & 1 & 1.28 & 2.12 & 1.70 & $\begin{array}{l}\text { Interleukin-4 induced protein } 1 \text { precursor (FIG-1 protein; mFIG1). } \\
\text { [Source: Uniprot/SWISSPROT: Acc: O09046] }\end{array}$ \\
\hline & 0.035 & 1 & 0.42 & 0.44 & 0.60 & $\begin{array}{l}\text { Interleukin-6 precursor (IL-6; Interleukin HP-1; B-cell hybridoma } \\
\text { growth factor). [Source: Uniprot/SWISSPROT; Acc: P08505] }\end{array}$ \\
\hline Other proteins & 0.036 & 1 & 2.73 & 2.74 & 1.74 & 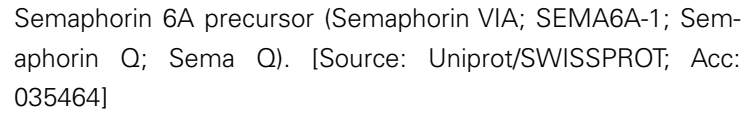 \\
\hline
\end{tabular}

Fold changes across all time points are normalized to $\mathrm{E} 10 \mathrm{H}$.

hypoxia were not studied. Their results demonstrated that in the absence of VEGF-A/C, E27 normoxic EBs was capable of forming both blood and lymphatic structures, however, adding VEGF-A or VEGF-C to the media further potently promoted lymphatic vessel formation. In our study, upon addition of growth factors, E22.5 normoxic EBs (N + VEGF-A/C) formed MECA-32+ blood vessel-like structures similar to those seen by Liersch et al. (2006). However organized lymphatic structures were lacking at this stage. One explanation for this disparity could be that Liersch et al. (2006) used twice the concentration of VEGF-C compared to our study. This difference is significant in light of the fact that a more dominant effect was observed after the addition of VEGF-C than with VEGF-A alone. Furthermore Kreuger et al. (2006) confirmed that a strong lymphangiogenic effect was not observed by VEGF-A alone. Likewise other studies have documented a strong synergism between VEGF-A and VEGF-C induction of lymphangiogenesis in cultured rat LECs (Whitehurst et al., 2006). Hence it is plausible that a synergistic effect between VEGF-A and VEGF$\mathrm{C}$ triggers the commitment of BECs and LECs toward vessel formation.

Hypoxia is a major physiological inducer of VEGF-A expression (Shweiki et al., 1992). Recent findings (Han et al., 2010) have determined a novel mechanism via HIF-1 $\beta$ by which hypoxia is capable of modulating the temporal expression of VEGF receptors, 
Table 3 | The raw normalized microarray data for selected lymphatic markers at different ages (E10-E20) and oxygen treatments are given.

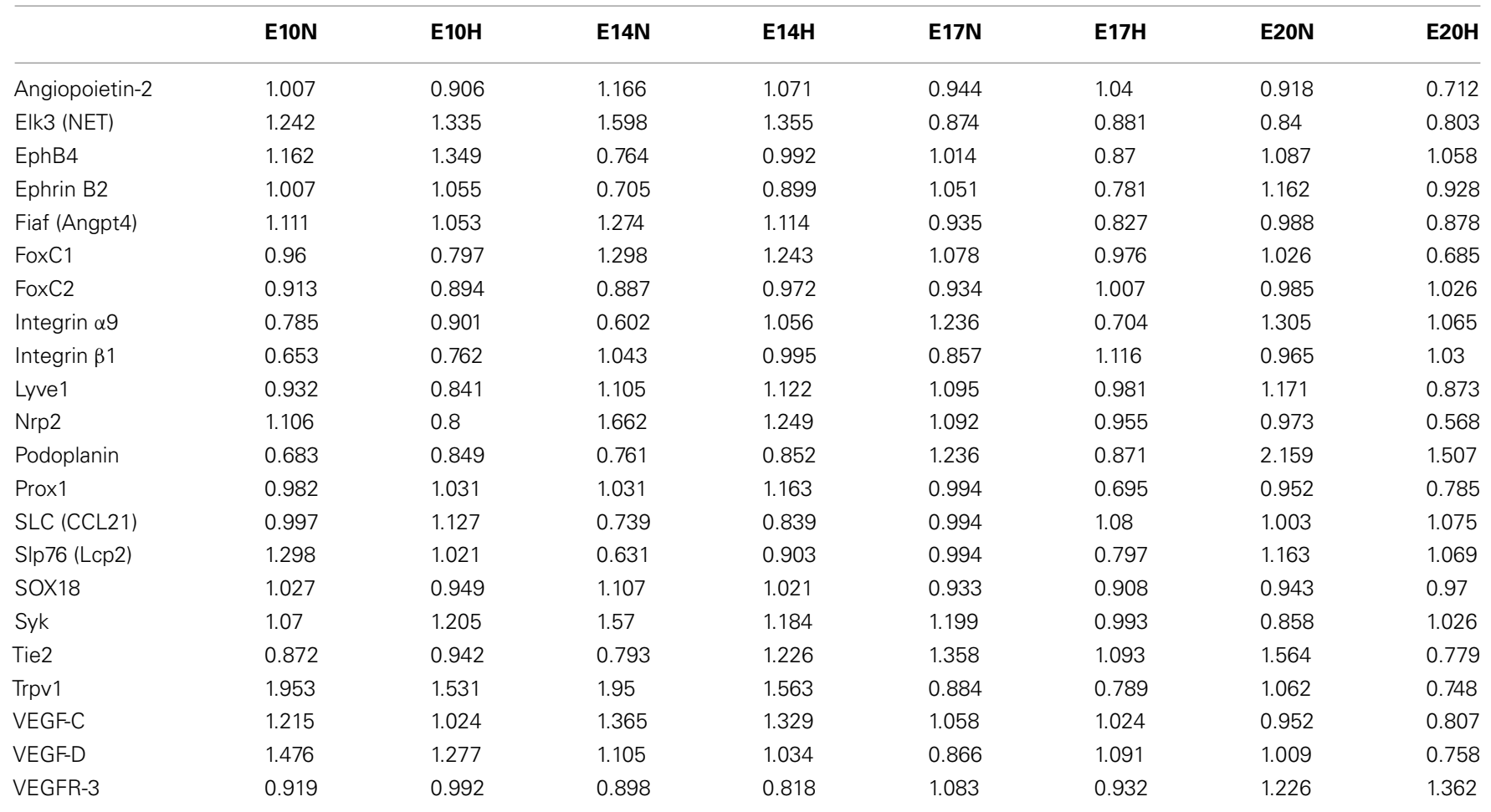

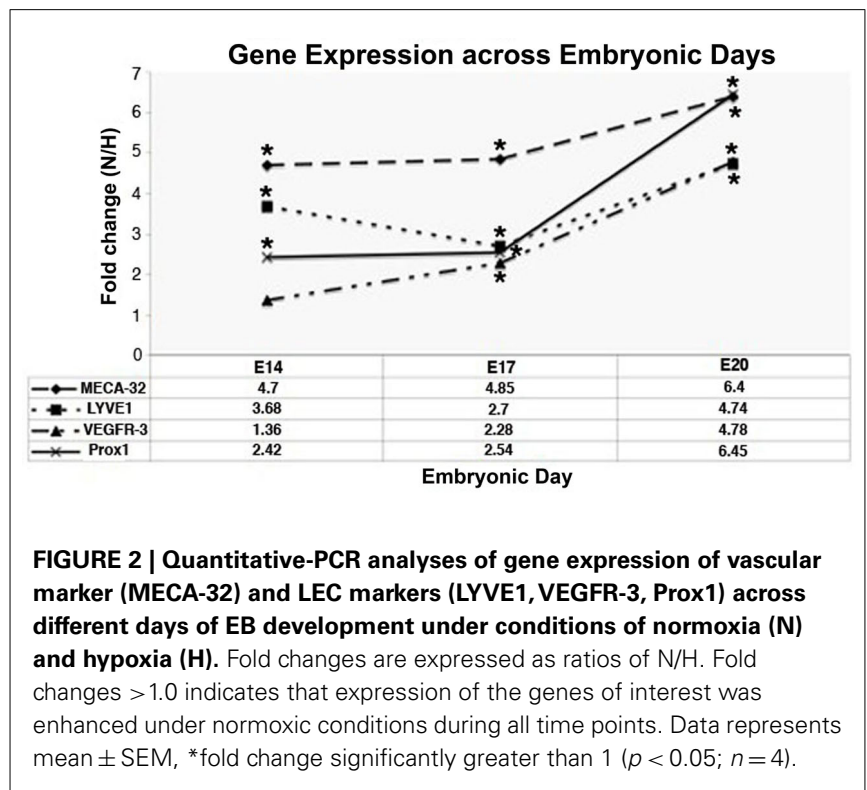

which are consequently known to be critical for the formation of vessel networks. Our microarray and qPCR data (Table 2 and Figure 2, respectively) corroborate well with the IHC data (Figure 3) showing normoxic EBs committed toward both blood $\left(\right.$ MECA-32 $^{+}$) and lymphatic (Prox1 ${ }^{+}$) cell lineages prior to E22.5. However normoxic cultures failed to form either blood or lymphatic vessel-like structures, indicating that these EBs have the necessary potential but lack the guidance mechanism via VEGFR1 and VEGF-A to allow the more structured blood or lymphatic

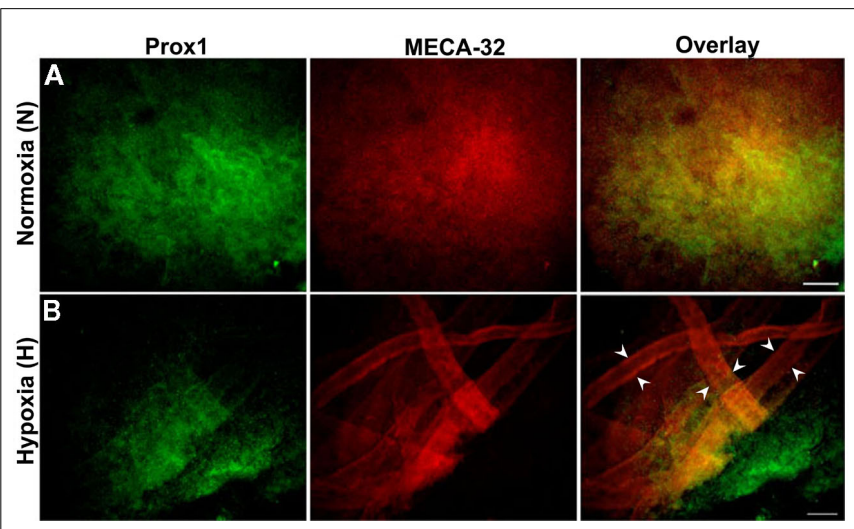

FIGURE 3 | Double immunofluorescence staining of E22.5-day-old EBs. Representative results from three different experiment sets for each marker genes are shown. Lymphatic ECs (LECs) and vascular ECs (BECs) are identified with Prox1 (green) and MECA-32 (red) markers respectively. EBs were grown under normoxic or hypoxic conditions as described in the Section "Materials and Methods." (A) Normoxic EBs allowed differentiation of both BECs and LECs but failed to form vessel-like structures. (B) Hypoxia alone induces a network of blood vessel-like structures, indicated by arrowheads and early differentiation of LECs. Images were taken at $40 x$ magnification (scale bar $30 \mu \mathrm{m}$ ).

vessel formation. Conversely, hypoxic EBs had adequate VEGFR-1 and VEGF-A expression prior to E22.5, thus allowing angiogenesis to occur, indicated by the presence of MECA- $32^{+}$blood vessel-like structures (Figure 3). These blood vessel-like structures formed despite the fact that MECA-32 expression was at least sixfold less (Figure 2) than normoxic cultures, suggesting a need for the timely 


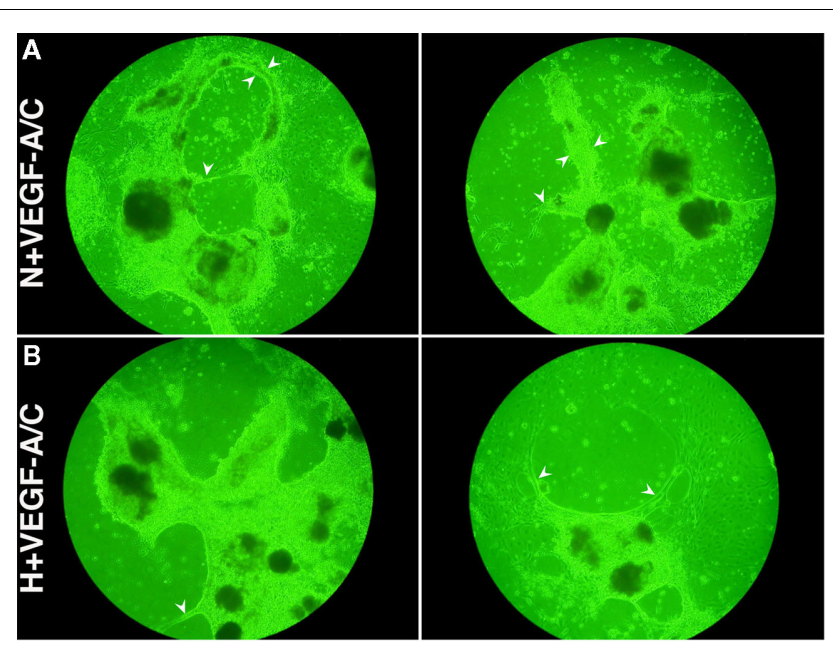

FIGURE 4 | Digital images (without magnification) of EBs at E22.5 grown under (A) N + VEGF-A/C (VN) and (B) H + VEGF-A/C (VH) treatments reveal contact of adjacent EBs to form cord-like structures, as marked by arrowheads. Representative result from 4 different experiment sets is shown.

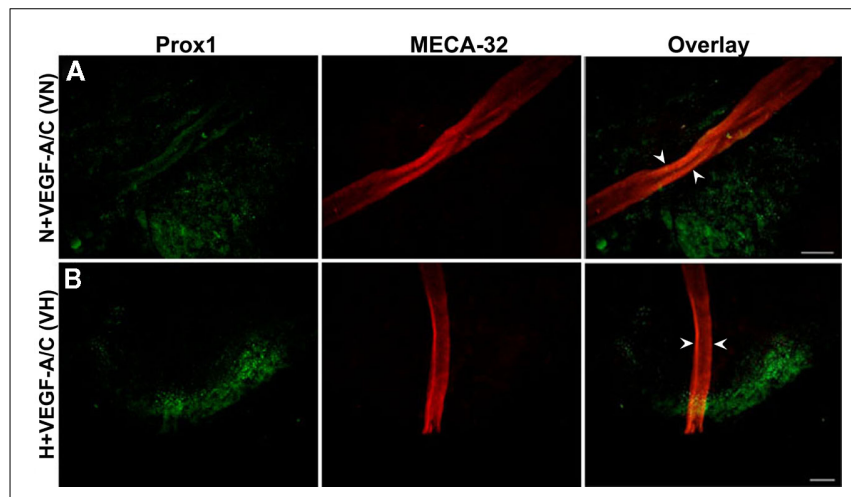

FIGURE 5 | Double immunofluorescence staining of E22.5-day-old EBs in the presence of VEGF-A and VEGF-C. Representative images from three different experiments sets for each marker genes are shown. Arrowheads indicate MECA-32 positive blood vessel-like structures in E22.5-day-old EBs grown under both VN (A) and VH (B) treatments. Prox1 positive LECs are in close proximity to the blood vessel-like structures. Images were taken at $40 \times$ magnification (scale bar $30 \mu \mathrm{m}$ ).

presence of guidance molecules, such as Angiopoietin-2 (Gale et al., 2002; Veikkola and Alitalo, 2002) and EphrinB2 (Adams et al., 1999; Makinen et al., 2005) for morphogenetic patterning events.

Kreuger et al. (2006) demonstrated that E22 EBs that were cultured on 3D collagen matrices and supplemented with VEGF-A/C growth factors, failed to develop lymphatic structures after being exposed to 8 days of hypoxia. Our results are in partial agreement with these findings since E22.5 hypoxic EBs (Figure 3B) in the absence of growth factors or a collagen-I matrix predominantly formed blood vessel-like structures, but not lymphatic vessel-like structures. This finding has also been recently

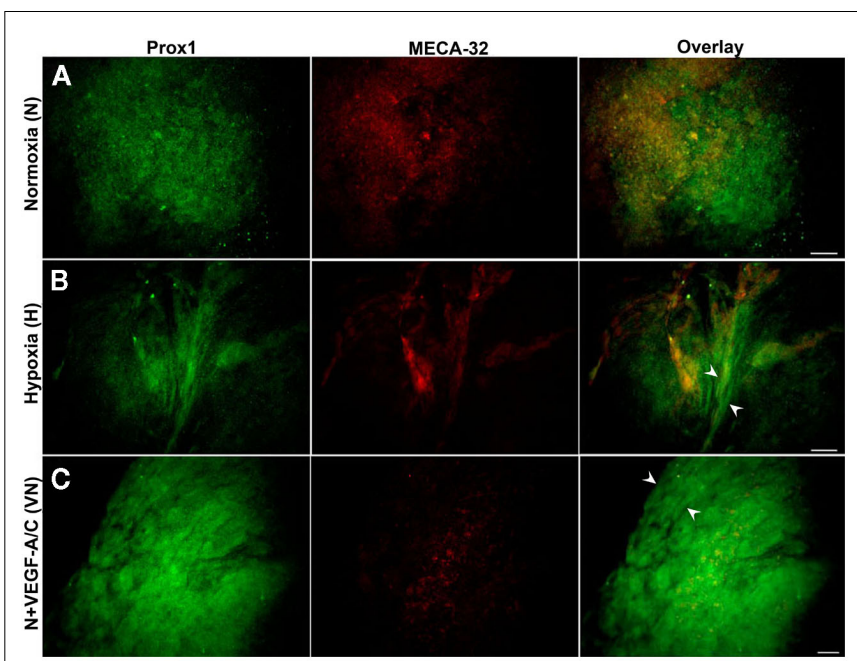

FIGURE 6 | Double immunostaining of E36.5-day-old EBs grown on collagen matrices subjected to three treatment types $(\mathrm{N}, \mathrm{H}, \mathrm{VN})$. Representative images from three different experiments sets for each marker genes are shown. (A) Normoxic EBs exhibited similar morphology to that observed at E22.5, illustrating differentiation of both BECs $\left(\mathrm{MECA}-32^{+}\right)$and LECs $\left(\operatorname{Prox}{ }^{+}\right)$. (B) Hypoxia alone induces some lymphatic vessel-like formation, as marked by arrowheads. (C) Normoxic EBs treated with VEGF-A/C predominantly induces lymphatic vessel-like structures (arrowheads). Images were taken at $40 \times$ magnification (scale bar $30 \mu \mathrm{m}$ ).

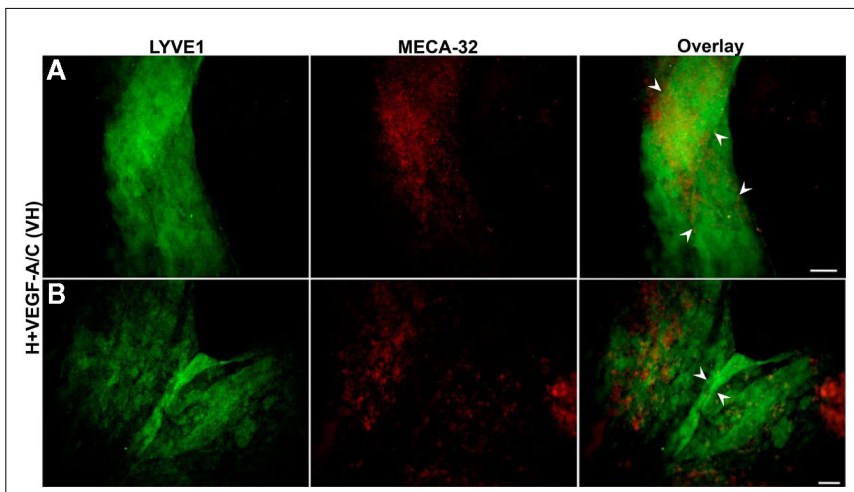

FIGURE 7 | Double immunofluorescent staining of E36.5-day-old hypoxic EBs grown on collagen-I coverslips and treated with VEGF-A/C. Representative images from three different experiments sets for each marker genes are shown. (A,B) Collagen-I promotes predominantly the organization of LYVE1 positive lymphatic vessel-like structures (marked by arrowheads) when stimulated under hypoxic and VEGF-A/C environments. There are some traces of MECA-32 positive BECs along with these lymphatic vessel-like structures. Unlike E22.5-day-old EBs, all four types of treatment at E36.5 failed to form organized blood vessel-like structures. Images were taken at $40 \times$ magnification (scale bar $30 \mu \mathrm{m}$ ).

supported by Han et al. (2010), wherein they demonstrated that hypoxia can promote EB vascular development independent of VEGF. However hypoxia itself, in the absence or presence of VEGF-A/C (Figures 6B and 7) stimulated the formation of a few lymphatic vessel-like structures from EBs grown on collagen matrices, but no blood vessel structures at E36.5. This mechanism 
is particularly relevant in the setting of tumor metastasis, where effects of hypoxia are observed on VEGF-C induced lymphangiogenesis (Schoppmann et al., 2006; Liang et al., 2008). Hence our data suggest that a combination of factors including collagen-I and VEGF-A/C helped toggle the switch from an initially predominant angiogenic to a lymphangiogenic process. In comparison, EBs grown on laminin alone (Figure 8) failed to form either distinctive blood or lymphatic structures, although they allowed BEC and LEC differentiation. This is consistent with findings documented from previous studies (Davis and Senger, 2005) that laminin blocks EC sprouting, but promotes EC differentiation. Further support for our findings was provided by Jakobsson et al. (2008) in which they demonstrated that although the composition of the vascular basement membrane in a murine EB model consisted of fibronectin, collagen and laminin, the knockdown of laminin alone from ES cells still supported the formation of vascular structures.

Collectively, our data demonstrate that while hypoxia alone promotes angiogenic processes, in the presence of collagen matrices lymphangiogenesis is favored in the mouse EB model at a later developmental time point. Furthermore, VEGF-A and VEGF-C induce both blood and lymphatic vessel-like structures during the differentiation of EBs. Thus our data corroborate well with other studies on lymphangiogenesis in mouse EB model (Nilsson

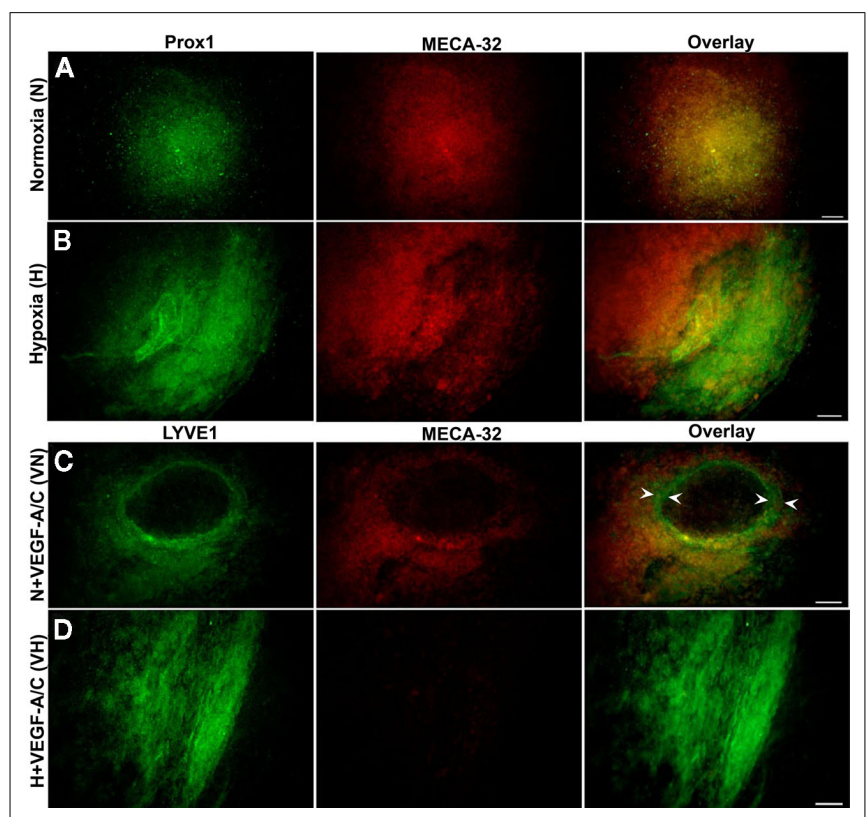

FIGURE 8 | E36.5-day-old EBs grown on laminin. EBs did not form vessel-like structures when stimulated under normoxic, hypoxic and VEGF-A/C environments. Representative data from three different experiments sets for each marker genes are shown. (A) Normoxic EBs grown on laminin did not show any distinguishable LEC phenotype, similar to those grown on collagen-I. (B) Hypoxic EBs did not induce any vessel-like structures within differentiating EBs. (C) N + VEGF-A/C EBs formed few ring-like structures (arrowheads) within the core of the EB and showed staining for LYVE1 in certain regions. (D) $\mathrm{H}+\mathrm{VEGF-A/C}$ environments promoted LEC $\left(\mathrm{LYVE}^{+}{ }^{+}\right.$) differentiation mostly on the periphery of differentiating EBs take. Images were en at $40 \times$ magnification (scale bar $30 \mu \mathrm{m})$. et al., 2004; Kreuger et al., 2006; Liersch et al., 2006). However given the fact that in vivo developmental processes are multifaceted in nature in comparison to any in vitro models, using the mouse EB model to study lymphatic vessel formation has several limitations. As seen in Figure 9, the whole-mount mesenteric preparation from a 3-month-old adult mouse exhibits a complex network of lymphatic and blood vessel structures. Both vascular and lymphatic systems show the presence of their respective EC markers in association with vascular actin positive muscle cells. Furthermore, the existence of initial lymphatics (ECs only) and collecting lymphatics (with valves and presence of muscle cells) are quite evident in the adult preparation. Though the mouse EB model could recapitulate early lymphangiogenic events, the intricate organization of the lymphatic system would be more difficult to mimic with this system. Nevertheless, the mouse EB paradigm develops lymphatic vessel-like structures in this study and in those shown by others (Nilsson et al., 2004; Kreuger et al., 2006; Liersch et al., 2006). It should also be noted that advanced $3 \mathrm{D}$ reconstruction images are warranted to further validate the vessel-like structures presented in this study. Nevertheless, the event occurs only at a much later time point of EB development, about E22-E36.5. These data clearly indicate that there are several additional factors likely to be involved and that the timing of various stimuli has to be coordinated in differentiating EBs to mimic conditions that occur in vivo. For example, Marino et al. (2011) have recently shown that retinoic acid signaling plays an essential role in the earliest stages of lymphangiogenesis using mouse EB model. In addition, having the knowledge through molecular studies and knockout models of the relevance of several genes, for example, FoxC2 for valve formation (Dagenais et al., 2004; Petrova et al., 2004), Angiopoietin-2 (Gale et al., 2002; Veikkola and Alitalo, 2002), and EphrinB2 (Adams et al., 1999; Makinen et al., 2005) for the patterning of the lymphatic system would

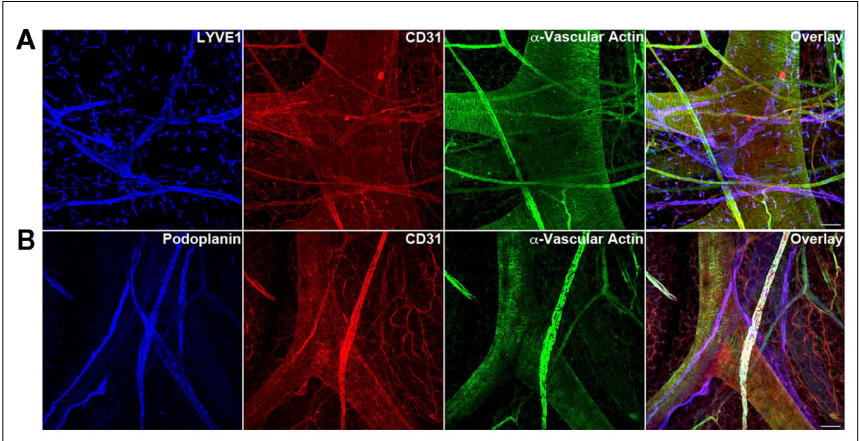

FIGURE 9 | Triple immunofluorescence staining (20x magnification) of whole-mount mesenteric preparations of 3-month adult mice visualizing lymphatic vessels using LEC markers (A) LYVE1 (B) podoplanin and blood vessels using BEC marker CD31. Representative images from three different experiments sets for each marker genes are shown. Muscle cells are identified with $\alpha$-vascular actin. The adult mouse mesentery demonstrates a complex network of blood and lymphatic vessels of different sizes. Initial lymphatic vessels (LYVE1+/Podoplanin ${ }^{+}$) are devoid of muscle cells ( $\alpha$-vascular actin ${ }^{-}$) whereas collecting lymphatic vessels (LYVE1-/Podoplanin ${ }^{+}$) show a nice arrangement of muscle cells $\left(\alpha\right.$-vascular $\left.\operatorname{actin}^{+}\right)$. Images were taken at $20 \times$ magnification (scale bar $12 \mu \mathrm{m})$. 
be greatly beneficial in applying the same information in the EB model to understand the complex mechanisms of lymphatic vessel development.

\section{REFERENCES}

Adams, R. H., Wilkinson, G. A., Weiss, C., Diella, F., Gale, N. W., Deutsch, U., Risau, W., and Klein, R. (1999). Roles of ephrinB ligands and EphB receptors in cardiovascular development: demarcation of arterial/venous domains, vascular morphogenesis, and sprouting angiogenesis. Genes Dev. 13, 295-306.

Banerji, S., Ni, J., Wang, S. X., Clasper, S., Su, J., Tammi, R., Jones, M., and Jackson, D. G. (1999). LYVE-1, a new homologue of the CD44 glycoprotein, is a lymph-specific receptor for hyaluronan. J. Cell Biol. 144, 789-801.

Borisov, A. (2005). Functional anatomy of lymphangion. Morfologiia 128, $18-27$.

Cueni, L. N., Chen, L., Zhang, H., Marino, D., Huggenberger, R., Alitalo, A., Bianchi, R., and Detmar, M. (2010) Podoplanin-Fc reduces lymphatic vessel formation in vitro and in vivo and causes disseminated intravascular coagulation when transgenically expressed in the skin. Blood 116, 4376-4384.

Dagenais, S. L., Hartsough, R. L., Erickson, R. P., Witte, M. H., Butler, M. G., and Glover, T. W. (2004). Foxc2 is expressed in developing lymphatic vessels and other tissues associated with lymphedema-distichiasis syndrome. Gene Expr. Patterns 4, 611-619.

Davis, G. E., and Senger, D. R. (2005) Endothelial extracellular matrix: biosynthesis, remodeling, and functions during vascular morphogenesis and neovessel stabilization. Circ. Res. 97, 1093-1107.

Dumont, D. J., Jussila, L., Taipale, J., Lymboussaki, A., Mustonen, T., Pajusola, K., Breitman, M., and Alitalo, K. (1998). Cardiovascular failure in mouse embryos deficient in VEGF receptor-3. Science 282, 946-949.

Ezekiel, U. (2007). Single embryoid body formation in a multi-well plate. Electron. J. Biotechnol. 10, 328-335.

Gale, N. W., Thurston, G., Hackett, S. F., Renard, R., Wang, Q., McClain, J., Martin, C., Witte, C., Witte, M. H., Jackson, D., Suri, C., Campochiaro, P. A., Wiegand, S. J., and Yancopoulos, G. D. (2002). Angiopoietin2 is required for postnatal angiogenesis and lymphatic patterning, and only the latter role is rescued by Angiopoietin-1. Dev. Cell 3, 411-423.

Gashev, A. A., Davis, M. J., Delp, M. D., and Zawieja, D. C. (2004). Regional variations of contractile activity in isolated rat lymphatics. Microcirculation 11, 477-492.

Gashev, A. A., Davis, M. J., and Zawieja, D. C. (2002). Inhibition of the active lymph pump by flow in rat mesenteric lymphatics and thoracic duct. J. Physiol. (Lond.) 540, 1023-1037.

Han, Y., Kuang, S.-Z., Gomer, A., and Ramirez-Bergeron, D. L. (2010). Hypoxia influences the vascular expansion and differentiation of embryonic stem cell cultures through the temporal expression of VEGF-receptors in an ARNTdependent manner. Stem Cells 28, 799-809.

Hong, Y. K., Harvey, N., Noh, Y. H., Schacht, V., Hirakawa, S., Detmar, M., and Oliver, G. (2002). Prox1 is a master control gene in the program specifying lymphatic endothelial cell fate. Dev. Dyn. 225, 351-357.

Hyde, J. A., Trzeciakowski, J. P., and Skare, J. T. (2007). Borrelia burgdorferi alters its gene expression and antigenic profile in response to $\mathrm{CO} 2$ levels. J. Bacteriol. 189, 437-445.

Jakobsson, L., Domogatskaya, A., Tryggvason, K., Edgar, D., and ClaessonWelsh, L. (2008). Laminin deposition is dispensable for vasculogenesis but regulates blood vessel diameter independent of flow. FASEB J. 22, 1530-1539.

Jeltsch, M., Kaipainen, A., Joukov, V., Meng, X., Lakso, M., Rauvala, H., Swartz, M., Fukumura, D., Jain, R. K., and Alitalo, K. (1997). Hyperplasia of lymphatic vessels in VEGFC transgenic mice. Science 276, 1423-1425.

Kaipainen, A., Korhonen, J., Mustonen, T., van Hinsbergh, V. W., Fang, G. H., Dumont, D., Breitman, M., and Alitalo, K. (1995). Expression of the fms-like tyrosine kinase 4 gene becomes restricted to lymphatic endothelium during development. Proc. Natl. Acad. Sci. U.S.A. 92, 3566-3570.

Karkkainen, M. J., Haiko, P., Sainio, K., Partanen, J., Taipale, J., Petrova, T. V., Jeltsch, M., Jackson, D. G., Talikka, M., Rauvala, H., Betsholtz, C., and Alitalo, K. (2004). Vascular endothelial growth factor $\mathrm{C}$ is required for sprouting of the first lymphatic vessels from

\section{ACKNOWLEDGMENTS}

This work was supported by National Institutes of Health grants HL-80526 and KO2 HL-86650.

embryonic veins. Nat. Immunol. 5, 74-80.

Kreuger, J., Nilsson, I., Kerjaschki, D., Petrova, T., Alitalo, K., and ClaessonWelsh, L. (2006). Early lymph vessel development from embryonic stem cells. Arterioscler. Thromb. Vasc. Biol. 26, 1073-1078.

Liang, X., Yang, D., Hu, J., Hao, X. Gao, J., and Mao, Z. (2008). Hypoxia inducible factor-alpha expression correlates with vascular endothelial growth factor-C expression and lymphangiogenesis/angiogenesis in oral squamous cell carcinoma. Anticancer Res. 28, 1659-1666.

Liersch, R., Nay, F., Lu, L., and Detmar, M. (2006). Induction of lymphatic endothelial cell differentiation in embryoid bodies. Blood 107, 1214-1216.

Makinen, T., Adams, R. H., Bailey, J., Lu, Q., Ziemiecki, A., Alitalo, K., Klein, R., and Wilkinson, G. A. (2005). $\mathrm{PDZ}$ interaction site in ephrinB2 is required for the remodeling of lymphatic vasculature. Genes Dev. 19, 397-410.

Marino, D., Dabouras, V., Brandli, A. W., and Detmar, M. (2011). A role for all-trans-retinoic acid in the early steps of lymphatic vasculature development. J. Vasc. Res. 48, 236-251.

Muthuchamy, M., Gashev, A., Boswell, N., Dawson, N., and Zawieja, D. (2003). Molecular and functional analyses of the contractile apparatus in lymphatic muscle. FASEB J. 17 , 920-922.

Navarro, A., Perez, R. E., Rezaiekhaligh, M., Mabry, S. M., and, Ekekezie, II. (2008). T1alpha/podoplanin is essential for capillary morphogenesis in lymphatic endothelial cells. Am. J. Physiol. Lung Cell. Mol. Physiol. 295, L543-L551.

Nilsson, I., Rolny, C., Wu, Y., Pytowski, B., Hicklin, D., Alitalo, K., ClaessonWelsh, L., and Wennstrom, S. (2004). Vascular endothelial growth factor receptor-3 in hypoxia-induced vascular development. FASEB J. 18 1507-1515.

Oliver, G. (2004). Lymphatic vasculature development. Nat. Rev. Immunol. 4, 35-45.

Oliver, G., and Alitalo, K. (2005) The lymphatic vasculature: recent progress and paradigms. Annu. Rev. Cell Dev. Biol. 21, 457-483.

Petrova, T. V., Karpanen, T., Norrmen, C., Mellor, R., Tamakoshi, T., Finegold, D., Ferrell, R., Kerjaschki,
D., Mortimer, P., Yla-Herttuala, S., Miura, N., and Alitalo, K. (2004). Defective valves and abnormal mural cell recruitment underlie lymphatic vascular failure in lymphedema distichiasis. Nat. Med. 10, 974-981.

Rockson, S. G. (2006). Lymphedema. Curr. Treat. Options Cardiovasc. Med. 8, 129-136.

Sabin, F. (1902). On the origin of the lymphatic system from the veins, and the development of lymph hearts and thoracic duct in the pig. Am. J. Anat. 1, 367-389.

Schacht, V., Ramirez, M. I., Hong, Y. K., Hirakawa, S., Feng, D., Harvey, N., Williams, M., Dvorak, A. M., Dvorak, H. F., Oliver, G., and Detmar, M. (2003). T1alpha/podoplanin deficiency disrupts normal lymphatic vasculature formation and causes lymphedema. EMBO J. 22, 3546-3556.

Schmid-Schonbein, G. (1990) Microlymphatics and lymph flow. Physiol. Rev. 70, 987-1028.

Schoppmann, S. F., Fenzl, A., Schindl, M., Bachleitner-Hofmann, T., Nagy, K., Gnant, M., Horvat, R., Jakesz, R., and Birner, P. (2006). Hypoxia inducible factor-1alpha correlates with VEGF-C expression and lymphangiogenesis in breast cancer. Breast Cancer Res. Treat. 99, 135-141.

Shweiki, D., Itin, A., Soffer, D., and Keshet, E. (1992). Vascular endothelial growth factor induced by hypoxia may mediate hypoxiainitiated angiogenesis. Nature 359, 843-845.

Szuba, A., and Rockson, S. G. (1998) Lymphedema: classification, diagnosis and therapy. Vasc. Med. 3, 145-156.

Veikkola, T., and Alitalo, K. (2002) Dual role of Ang2 in postnatal angiogenesis and lymphangiogenesis. Dev. Cell 3, 302-304

Whitehurst, B., Eversgerd, C., Flister, M., Bivens, C. M., Pickett, B., Zawieja D. C., and Ran, S. (2006). Molecular profile and proliferative responses of rat lymphatic endothelial cells in culture. Lymphat. Res. Biol. 4, 119-142.

Wigle, J. T., Harvey, N., Detmar, M., Lagutina, I., Grosveld, G., Gunn, M. D., Jackson, D. G., and Oliver, G. (2002). An essential role for Proxl in the induction of the lymphatic endothelial cell phenotype. EMBO J. 21, 1505-1513. 
Wigle, J. T., and Oliver, G. (1999) Proxl function is required for the development of the murine lymphatic system. Cell 98, 769-778.

Yuan, L., Moyon, D., Pardanaud, L., Breant, C., Karkkainen, M. J., Alitalo, K., and Eichmann, A. (2002). Abnormal lymphatic vessel development in neuropilin 2 mutant mice. Development 129, 4797-4806.
Conflict of Interest Statement: The authors declare that the research was conducted in the absence of any commercial or financial relationships that could be construed as a potential conflict of interest.

Received: 27 May 2011; accepted: 30 November 2011; published online: 20 December 2011.
Citation: Foskett AM, Ezekiel UR, Trzeciakowski JP, Zawieja DC and Muthuchamy $M$ (2011) Hypoxia and extracellular matrix proteins influence angiogenesis and lymphangiogenesis in mouse embryoid bodies. Front. Physio. 2:103. doi: 10.3389/fphys.2011.00103

This article was submitted to Frontiers in Vascular Physiology, a specialty of Frontiers in Physiology.
Copyright (C) 2011 Foskett, Ezekiel, Trzeciakowski, Zawieja and Muthuchamy. This is an open-access article distributed under the terms of the Creative Commons Attribution Non Commercial License, which permits non-commercial use, distribution, and reproduction in other forums, provided the original authors and source are credited. 